\title{
Ethno Veterinary Therapeutic Practices of Medicinal Flora in Livestock Health Care by Attappadi Tribal farmers of Kerala
}

Nisha A ( $\sim$ nishaaaravind25@gmail.com )

Vellore Institute of Technology: VIT University https://orcid.org/0000-0001-9347-9038

Vimal Rajkumar $\mathbf{N}$

TANUVAS: Tamil Nadu Veterinary and Animal Sciences University

\section{Research}

Keywords: Attappadi tribes, Consensus factor, Ethno Veterinary Medicinal plants, Livestock Health, Medicinal uses

Posted Date: March 4th, 2021

DOI: https://doi.org/10.21203/rs.3.rs-258368/v1

License: (c) (i) This work is licensed under a Creative Commons Attribution 4.0 International License. Read Full License 


\section{Abstract}

Background- Attappadi is the largest tribal settlement area in Kerala with three tribes viz., Irulas, Mudugas and Kurumbas who are traditionally engaged in livestock activities. The present study was undertaken to document the different plants of ethnoveterinary uses and to identify the most prevailing livestock disease category among livestock farmers of Attappadi tribal area. Hence this study was designed and conducted to understand and effectively use the knowledge, and for the proper selection of important plant species for the most prevailing illness.

Methods- This study was conducted in the period of December 2018 - April 19 using a structured interview with tribal farmers, EVM healers and key informants engaged in the livestock farming.

Results- The present study documented 55 plant species under 39 families for treatment of 15 categories of ailments with their dosages and parts used in Attappadi tribal area. There was great agreement among the informants regarding ethno veterinary uses of plants with Factor of Informants Consensus $\left(F_{I C}\right)$ value ranging from 0.50 to 0.97 , with an average value of 0.87 .

Conclusion- The study reports 55 medicinal plants with their uses and dosage along with the highlighted high $\mathrm{F}_{\mathrm{IC}}$ values indicates that the species traditionally used to treat these ailments are worth searching for bioactive compounds and this has illuminated the cultural importance of this ethno veterinary wisdom which is consonantly blended with the tribal culture of the area.

\section{Introduction}

Indian economy and its population depend greatly on agriculture and the livestock. Livestock contributes $4.11 \%$ GDP and $25.6 \%$ of total Agriculture GDP. About 20.5 million people depend upon livestock for their livelihood. Livestock provides livelihood to two-third of rural community $[1,2,3]$. Hence the diseases in livestock should be given more importance owing to their significant role in micro and macro economy of nation. Ethnic communities keep their livestock as a symbol of heritage and pride, and they follow indigenous practices to treat their animals. Knowledge on traditional and indigenous practices has been transferred orally over ethnic generations but under the semblance of civilization this knowledge is under great threat of cultural extinction [4]. About $80 \%$ of people in the developing world use natural remedies and traditional plant-based medicines for their primary healthcare [5]. Tribal communities are the torch bearers of the Ethnoveterinary knowledge. In Kerala, there are 36 tribal communities consist of a total population 4, 84,839 [6]. Palakkad, one among the largest tribal concentrated districts of Kerala has 10.1 per cent of the total tribal population of the state. Attappadi is the largest tribal settlement area of 
Palakkad district with three tribes viz., Irulas (84 per cent), Mudugas (10 per cent) and Kurumbas (six per cent) who are traditionally engaged in livestock activities [7, 8]. Hence this study was designed and conducted to understand and effectively use this knowledge, and the proper selection of important plant species for the most prevailing illness must be determined.

\section{Materials And Methods}

The present study was conducted purposively in Attappadi block of Palakkad district in Kerala as this is one among the largest tribal settlements with considerable livestock population. Attappadi block comprised of three panchayats, Agali, Pudur and Sholayoor (Fig. 1). A total of 40 tribal livestock farmers from each of the three panchayats, thus a total of 120 tribal livestock farmers were selected randomly as respondents for the study. Ethno veterinary medicine healers and key informants were interviewed along with the tribal farmers to gather information on EVM practises. Prior Informed Consent (PIC) was taken from the Knowledge Providers. A descriptive research design was employed. Personal interviews by pretested interview schedule and Participatory Rural Appraisal (PRA) approach was used to serve the purpose of data collection ((Fig. 2). During the field study, information on uses of plants to treat different illnesses of livestock, parts used, modes of preparation, dosage and administration of medicine have been collected. Based on the information obtained from the informants in the study area, all the reported ailments have been grouped into 15 categories.

The level of homogeneity among information provided by different informants was calculated by the Informants' Consensus Factor, $\mathrm{F}_{\mathrm{IC}}[9]$ using the following formula:

$F_{I C}=$ Nur $-\mathrm{Nt} /($ Nur -1$)$ Where,

Nur = number of use reports from informants for a particular plant-use category

$\mathrm{Nt}=$ number of taxa or species that are used for that plant use category for all informants.

Here in this study each polyherbal remedy has been considered as single use-report. The value of $F_{I C}$ provides a range of 0 to 1 , where a higher value signifies its greater authenticity as a fixed group of plants are used frequently for that disease or disease category. On the other hand, lower value signifies

disagreement among the informants. The similar studies were conducted to know the consent of relevant ethnoveterinary practices in livestock. $[10,11,12]$.

\section{Results And Discussion}

In the present study the traditional EVM in treatment of different livestock ailments were recorded through the interactive discussions with the tribal farmers and traditional healers (Fig. 3). More than three fourth of the respondents (81.67\%) shared their knowledge on EVM. A minority (18.37\%) felt that the sharing of information would lead to the loss of healing potential of these plants. A total of 55 plant species under 39 families in Attappadi area have been documented for treatment of 51 categories of 
ailments. Factors for Informants' consensus $\left(F_{I C}\right)$ have been used to identify most potentially effective medicinal plant species as well as plant-based remedies used in the culture of rural people of the study area [10]. Here in this study each polyherbal remedy has been considered as single use-report.

As Table 1.1. bespeaks, there was a medium to high level of consensus among the informants in the study area. The $F_{I C}$ value for different disease categories has ranged from 0.50 to 0.97 with an average value of 0.87 that has indicated the level of agreement among the informants regarding different phyto therapeutic uses of medicinal plants. Similar findings were reported from Velliangiri holy hills [13].

Table 1.1

Categories of ailments and informant consensus factor (FIC) for each category

\begin{tabular}{|lllll|}
\hline Sl.no & Illness Category & $\mathbf{N}_{\mathbf{u r}}$ & $\mathrm{N}_{\mathbf{t}}$ & $\mathbf{F}_{\mathrm{IC}}$ \\
\hline 1. & Gastro-Intestinal disorders & 89 & 8 & 0.92 \\
\hline 2. & Poisoning & 9 & 3 & 0.75 \\
\hline 3. & Respiratory disorders & 42 & 4 & 0.92 \\
\hline 4. & Skin diseases & 48 & 7 & 0.87 \\
\hline 5. & Dewormer & 29 & 4 & 0.89 \\
\hline 6. & Fever, Cold & 46 & 4 & 0.93 \\
\hline 7. & Mastitis & 46 & 5 & 0.91 \\
\hline 8. & Reproductive disorders & 61 & 8 & 0.88 \\
\hline 9. & Acaricides & 28 & 2 & 0.96 \\
\hline 10. & Musculo Skeletal disorders & 19 & 3 & 0.88 \\
\hline 11. & FMD & 91 & 5 & 0.96 \\
\hline 12. & Pox & 76 & 3 & 0.97 \\
\hline 13. & Anti - Inflammatory & 5 & 3 & 0.50 \\
\hline 14. & HS & 4 & 2 & 0.67 \\
\hline 15. & Snake bite & 25 & 2 & 0.96 \\
\hline
\end{tabular}

Pox showed highest $F_{I C}$ value, 0.97 with 76 use reports for three plant species, which was followed by FMD (0.96) with 91 use reports for five plant species, ectoparasitism (0.96) with 28 use reports for two plant species, and snake bite (0.96) with 25 use reports for two plant species. High $F_{I C}$ values possibly showed that these ailments were common in the study area which could be attributed to the better care of farmers since theses disease categories had direct negative influence on production of the animals. Medicinal plants supposed to be efficient in treating ailments having high $\mathrm{F}_{\mathrm{IC}}$ value [14]. 
This was followed by gastro - intestinal disorders, respiratory disorders, mastitis, fever and cold with values ranged from 0.93 to 0.91 . The least agreement (low $F_{I C}$ value) between informants was observed for plants used to cure inflammatory conditions $(0.50)$ with 5 use reports for three plant species which could be due to the fact that those conditions were remained unnoticed by the farmers as they had no direct influence on animal's production [15]. Similar results were reported among the tribal farmers of Malda district of Bengal [16].

Different EVM practises have been identified and discussed under the following subheads

1. Gastrointestinal disorders - diarrhoea, constipation, indigestion, bloat and stomach pain

2. Respiratory ailments, fever and cold

3. General poisoning and snake venomation conditions

4. Skin diseases - Lacerated wounds, Umbilical wounds, warts, traumatic myasis,

5. Deworming and ectoparasiticides

6. Reproductive disorders - Infertility, anoestrum, retention of foetal membranes, uterine prolapsed, uterine infection, uterine involution and dystocia.

7. Musculoskeletal disorders - arthritis / joint pain, fracture and sprain.

8. Mastitis

9. Foot and Mouth Disease

10. Pox

11. Inflammatory conditions

12. Haemorrhagic septicaemia 
Table 1.2

Ethnoveterinary Medicinal Plants Utilized by Tribal Farmers of Attappadi to manage Livestock Gastro Intestinal Diseases

\begin{tabular}{|c|c|c|c|c|c|}
\hline \multicolumn{6}{|c|}{ Ailment/Disease: Gastro Intestinal Diseases } \\
\hline SI.no & $\begin{array}{l}\text { Vernacular name/ } \\
\text { Scientific name } \\
\text { (Family) }\end{array}$ & Parts used & Conditions & $\begin{array}{l}\text { Route of } \\
\text { administration }\end{array}$ & Dosage \\
\hline 1. & $\begin{array}{l}\text { Adaykka } \\
\text { Arecanut } \\
\text { Areca catechu } \\
\text { (Arecaceae) }\end{array}$ & Fruit & $\begin{array}{l}\text { Worm } \\
\text { infestation }\end{array}$ & $\begin{array}{l}\text { Orally as } \\
\text { pulverized or } \\
\text { bolus form }\end{array}$ & $50 \mathrm{~g}$ \\
\hline 2. & $\begin{array}{l}\text { Ajowan } \\
\text { Trachyspermum ammi } \\
\text { (Apiaceae) }\end{array}$ & Leaf, seed & Indigestion & $\begin{array}{l}\text { Orally mixed with } \\
\text { boiling water }\end{array}$ & $300 \mathrm{ml}$ \\
\hline 3. & $\begin{array}{l}\text { Betel } \\
\text { Piper betle (Piperaceae) }\end{array}$ & Seed & Indigestion & Oral mix & $30 \mathrm{~g}$ \\
\hline 4. & $\begin{array}{l}\text { Bitter gourd } \\
\text { Momordica charantia } \\
\text { (Cucurbitaceae) }\end{array}$ & Leaf & $\begin{array}{l}\text { Worm } \\
\text { infestation }\end{array}$ & Pulp as oral dose & $500 \mathrm{~g}$ \\
\hline 5. & $\begin{array}{l}\text { Coconut } \\
\text { Cocos nucifera } \\
\text { (Arecaceae) }\end{array}$ & $\begin{array}{l}\text { Flower, } \\
\text { tender water }\end{array}$ & Diarrhoea & Oral dose & $3-5 \mathrm{lr}$ \\
\hline 6. & $\begin{array}{l}\text { Curry leaves } \\
\text { Murraya koenigii } \\
\text { (Rutaceae) }\end{array}$ & Leaf & Stomach pain & $\begin{array}{l}\text { Ground and orally } \\
\text { given }\end{array}$ & $100 \mathrm{~g}$ \\
\hline 7. & $\begin{array}{l}\text { Fenugreek } \\
\text { Trigonella foenum- } \\
\text { graecum } \\
\text { (Leguminosae) }\end{array}$ & Seed, leaf & $\begin{array}{l}\text { Worm } \\
\text { infestation }\end{array}$ & Orally as paste & $\begin{array}{l}50-80 \\
\mathrm{~g}\end{array}$ \\
\hline 8. & $\begin{array}{l}\text { Garlic } \\
\text { Allium sativum L. } \\
\text { (Amaryllidaceae) }\end{array}$ & Clove & $\begin{array}{l}\text { Diarrhoea, } \\
\text { Indigestion }\end{array}$ & Orally as paste & $50 \mathrm{~g}$ \\
\hline
\end{tabular}




\section{Ailment/Disease: Gastro Intestinal Diseases}

\begin{tabular}{|c|c|c|c|c|c|}
\hline 9. & $\begin{array}{l}\text { Ginger } \\
\text { Zingiber officinale Rosc. } \\
\text { (Zingiberaceae) }\end{array}$ & Rhizome & $\begin{array}{l}\text { Constipation, } \\
\text { Indigestion }\end{array}$ & $\begin{array}{l}\text { Orally with other } \\
\text { ingredients }\end{array}$ & $100 \mathrm{~g}$ \\
\hline 10. & $\begin{array}{l}\text { Guava } \\
\text { Psidium guajava } \\
\text { (Myrtaceae) }\end{array}$ & Leaf & Bloat & Orally as paste & $150 \mathrm{~g}$ \\
\hline 11. & $\begin{array}{l}\text { Jeerakam } \\
\text { Cuminum cyminum } \\
\text { (Umbelliferae) }\end{array}$ & Fruit & Constipation & Orally & $25 \mathrm{~g}$ \\
\hline
\end{tabular}

$\begin{array}{lll}\text { 12. Kaayam } & \text { Resin Indigestion } & \begin{array}{l}\text { Orally mixed with } \\ \text { boiling water }\end{array} \\ \text { Asafoetida } & & \mathrm{g}\end{array}$

Ferula asafetida L.

(Apiaceae)

$\begin{array}{llll}\text { 13. Lemon } & \text { Leaf, fruit } & \begin{array}{l}\text { Worm } \\ \text { infestation }\end{array} & \text { Orally }\end{array}$

(Rutaceae)

$\begin{array}{llll}\text { 14. Mukkutti Stem } & \text { Diarrhoea } & \mathrm{g}\end{array}$

Biophytum nervifolium

(Oxalidaceae)

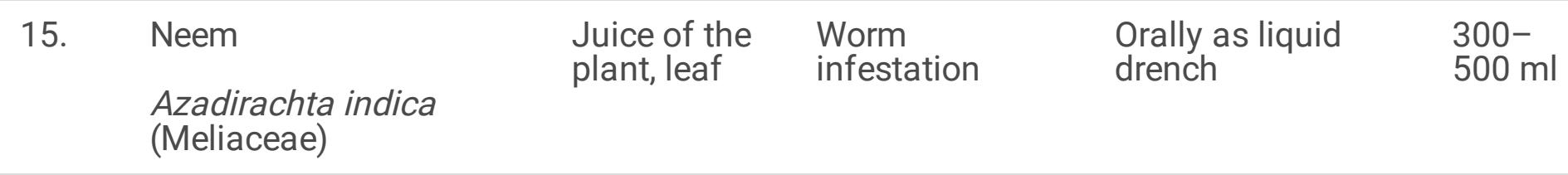

\begin{tabular}{|c|c|c|c|}
\hline $\begin{array}{l}\text { Papaya } \\
\text { Carica papaya }\end{array}$ & $\begin{array}{l}\text { Fruit, seed, } \\
\text { leaf }\end{array}$ & $\begin{array}{l}\text { Indigestion, } \\
\text { Worm } \\
\text { infestation }\end{array}$ & Orally as paste \\
\hline
\end{tabular}

(Caricaceae)

$\begin{array}{llll}\text { 17. Pepper } & \text { Seed, leaf Indigestion } & \text { Oral }\end{array}$

Piper nigrum L.

(Piperaceae) 


\begin{tabular}{|c|c|c|c|c|c|}
\hline \multicolumn{6}{|c|}{ Ailment/Disease: Gastro Intestinal Diseases } \\
\hline 18. & $\begin{array}{l}\text { Red chilly } \\
\text { Capsicum annum L. } \\
\text { (Solanaceae) }\end{array}$ & Fruit, stem & Constipation & Orally & $\begin{array}{l}15 \mathrm{no} / \\
30 \mathrm{~g}\end{array}$ \\
\hline 19. & $\begin{array}{l}\text { Red onion } \\
\text { Allium sepa L. } \\
\text { (Liliaceae) }\end{array}$ & Bulb & $\begin{array}{l}\text { Diarrhoea, } \\
\text { Indigestion }\end{array}$ & Orally as paste & $200 \mathrm{~g}$ \\
\hline 20. & $\begin{array}{l}\text { Shathavari } \\
\text { Asparagus racemosus } \\
\text { Wild (Liliaceae) }\end{array}$ & $\begin{array}{l}\text { Extracts } \\
\text { from dried } \\
\text { root }\end{array}$ & $\begin{array}{l}\text { Worm } \\
\text { infestation }\end{array}$ & Orally & $\begin{array}{l}100- \\
150 \mathrm{ml}\end{array}$ \\
\hline 21. & $\begin{array}{l}\text { Spinach } \\
\text { Spinacia oleracea } \\
\text { (Amaranthaceae) }\end{array}$ & Leaf, & $\begin{array}{l}\text { Worm } \\
\text { infestation }\end{array}$ & Orally as paste & $200 \mathrm{~g}$ \\
\hline 22. & $\begin{array}{l}\text { Tamarind } \\
\text { Tamarindus indica } \\
\text { (Fabaceae) }\end{array}$ & Leaf, fruit & Diarrhoea & Orally as paste & $100 \mathrm{~g}$ \\
\hline 23. & $\begin{array}{l}\text { Touch me not } \\
\text { Mimosa pudica } \\
\text { (Fabaceae) }\end{array}$ & $\begin{array}{l}\text { Leaf, root, } \\
\text { stem }\end{array}$ & Diarrhoea & $\begin{array}{l}\text { Paste is given } \\
\text { orally }\end{array}$ & $100 \mathrm{~g}$ \\
\hline 24. & $\begin{array}{l}\text { Turmeric } \\
\text { Curcuma domestica } \\
\text { vallars (Zingiberaceae) }\end{array}$ & Rhizome & Constipation & $\begin{array}{l}\text { Orally mixed with } \\
\text { boiling water }\end{array}$ & $100 \mathrm{~g}$ \\
\hline 25. & $\begin{array}{l}\text { Ungu } \\
\text { Milletia } \\
\text { Millettia pinnata } \\
\text { (Fabaceae) }\end{array}$ & $\begin{array}{l}\text { Root, stem, } \\
\text { bark }\end{array}$ & Indigestion & $\begin{array}{l}\text { Orally mixed with } \\
\text { boiling water }\end{array}$ & $100 \mathrm{~g}$ \\
\hline
\end{tabular}

As Table 1.2. depicts butter milk with garlic paste, a combination of red onion, little tree plant, jaggery and coconut flower is ground in equal proportion and ground curry leaves were used by 66.32 per cent of the respondents to treat diarrhoea. This clearly indicated that the above practise were highly effective in treating diarrhoea. Leaves of touch me not mixed with water was recommended for diarrhoea by 8.16 per cent of the respondents and tamarind by 6.12 per cent of the respondents. Under the condition constipation, the documented practises were use of honey in warm water by 10.20 per cent of the 
respondents, and a mixture of $10 \mathrm{~g}$ turmeric, $25 \mathrm{~g}$ cumin, $100 \mathrm{~g}$ jaggery, $100 \mathrm{~g}$ ginger and 15 red chillies by 26.53 per cent of the respondents.

There were four practises documented for the indigestion condition in animals. They were use of raw papaya (3.06\%), ground mixture of ginger, garlic, pepper, asafoetida, and betel (16.33\%), a combination of ajowan and red onion (18.37\%), stem bark of milletia is boiled in water which was dried to one - third and was given to animal by 4.08 per cent of the respondents. Bloat was treated with ground guava leaves $(36.73 \%)$ and stomach pain was treated with ground curry leaves orally $(45.91 \%)$.

To deworm the animals, powdered arecanut, mixture of papaya seeds and sugar was given for 5 days in calf by 6.12 per cent of the respondents. Ground neem leaves (33.67\%), spinach leaves, lemon in sesame oil, fenugreek, neem oil for three days $(42.86 \%)$, pulp of ground bitter gourd leaves, thumba plant, papaya and asparagus were given to deworm the animals by 29.60 per cent of the respondents. Widely used ectoparasiticides were neem oil, calcium hydroxide, cassia and ceylon leaves by majority of the respondents (54.08\%). 
Table 1.3

Ethnoveterinary Medicinal Plants Utilized by Tribal Farmers of Attappadi to manage Livestock Reproductive Health

\begin{tabular}{|c|c|c|c|c|c|}
\hline \multicolumn{6}{|c|}{$\begin{array}{l}\text { Ailment/Disease: } \\
\text { Reproductive Health }\end{array}$} \\
\hline SI.no & $\begin{array}{l}\text { Vernacular name/ } \\
\text { Scientific name } \\
\text { (Family) }\end{array}$ & Parts used & Conditions & $\begin{array}{l}\text { Route of } \\
\text { administration }\end{array}$ & Dosage \\
\hline 1. & $\begin{array}{l}\text { Aloe vera } \\
\text { Aloe } \\
\text { barbadensis Mill. } \\
\text { (Asphodelaceae) }\end{array}$ & $\begin{array}{l}\text { Leaf, extract and latex } \\
\text { of plant skin }\end{array}$ & $\begin{array}{l}\text { Infertility, } \\
\text { Mastitis }\end{array}$ & Orally & $\begin{array}{l}4 \\
\text { hands }\end{array}$ \\
\hline 2. & $\begin{array}{l}\text { Bamboo } \\
\text { Bambusa } \\
\text { arundinacea (Retz.) } \\
\text { Wild. (Poaceae) }\end{array}$ & Young stem, leaves & $\begin{array}{l}\text { Retention of } \\
\text { foetal } \\
\text { membranes }\end{array}$ & Orally & $250 \mathrm{~g}$ \\
\hline 3. & $\begin{array}{l}\text { Banana } \\
\text { Musa paradisiacal } \\
\text { L. (Musaceae) }\end{array}$ & Plant juice, Fruit, leaf & Anoestrum & Orally & $300 \mathrm{~g}$ \\
\hline 4. & $\begin{array}{l}\text { Chaff flower } \\
\text { Achyranthes } \\
\text { aspera } L \text {. } \\
\text { (Amaranthaceae) }\end{array}$ & $\begin{array}{l}\text { Herb, leaves, seeds, } \\
\text { root flower (whole plant) }\end{array}$ & Dystocia & Externally & $200 \mathrm{~g}$ \\
\hline 5. & $\begin{array}{l}\text { Greater yam } \\
\text { Dioscorea alata } \\
\text { (Dioscoreaceae) }\end{array}$ & Tuber, Leaf & $\begin{array}{l}\text { Retention of } \\
\text { foetal } \\
\text { membranes }\end{array}$ & Orally & $500 \mathrm{~g}$ \\
\hline 6. & $\begin{array}{l}\text { Malabar kino } \\
\text { Pterocarpus } \\
\text { marsupium } \\
\text { (Fabaceae) }\end{array}$ & Bark, leaf & $\begin{array}{l}\text { Uterine } \\
\text { infection }\end{array}$ & Orally & $300 \mathrm{~g}$ \\
\hline 7. & $\begin{array}{l}\text { Mango } \\
\text { Mangifera indica } \\
\text { Linn. } \\
\text { (Anacardiaceae) }\end{array}$ & Leaf & $\begin{array}{l}\text { Retention of } \\
\text { foetal } \\
\text { membranes }\end{array}$ & Orally & $500 \mathrm{~g}$ \\
\hline
\end{tabular}




\begin{tabular}{|c|c|c|c|c|c|}
\hline \multicolumn{6}{|c|}{$\begin{array}{l}\text { Ailment/Disease: } \\
\text { Reproductive Health }\end{array}$} \\
\hline 8. & $\begin{array}{l}\text { Muringa } \\
\text { Moringa oleifera } \\
\text { Lamk. } \\
\text { (Moringaceae) }\end{array}$ & $\begin{array}{l}\text { Leaf, root, juice of the } \\
\text { stem }\end{array}$ & $\begin{array}{l}\text { Infertility, } \\
\text { dystocia }\end{array}$ & $\begin{array}{l}\text { Orally \& } \\
\text { Externally }\end{array}$ & $\begin{array}{l}300 \mathrm{~g} \\
- \\
500 \mathrm{~g}\end{array}$ \\
\hline 9. & $\begin{array}{l}\text { Njerinjil } \\
\text { Tribulus terrestris } \\
\text { (Zygophyllaceae) }\end{array}$ & Seed & $\begin{array}{l}\text { Uterine } \\
\text { prolapse }\end{array}$ & Orally & $250 \mathrm{~g}$ \\
\hline 10. & $\begin{array}{l}\text { Pineapple } \\
\text { Ananas comosus } \\
\text { (Bromeliaceae) }\end{array}$ & Fruit & $\begin{array}{l}\text { Retention of } \\
\text { foetal } \\
\text { membranes }\end{array}$ & Orally & $\begin{array}{l}3 \mathrm{no} / \\
1500 \mathrm{~g}\end{array}$ \\
\hline 11. & $\begin{array}{l}\text { Piranda } \\
\text { Cissus } \\
\text { quadrangularis } \\
\text { (Vitaceae) }\end{array}$ & Stem, leaf & Infertility & Orally & $500 \mathrm{~g}$ \\
\hline
\end{tabular}

A perusal of Table 1.3 indicates that the infertility conditions were treated with boiled egg in sesame oil and equi proportion of aloe vera (4 hands), drumstick leaves, curry leaves and bone setter plant by 13.27 per cent of the respondents. Banana leaves were used to treat anoestrum $(9.18 \%)$. Retention of foetal membranes was treated with unripened pineapple, mango leaves, stem of bamboo and greater yams' leaves by 83.67 per cent of the respondents which indicated that the practises were highly effective to treat retention of foetal membranes.

Puncture vine boiled water is given in uterine prolapsed condition (12.24 \%), ground Malabar kino's sap is taken to prepare starch gruel was given to ail uterine infection $(23.47 \%)$. Uterine involution was hastened by papaya fruit $(29.60 \%)$. To avoid dystocia, muringa leaves' extract was pasted over the vulva, and chaff flower was given by 37.76 per cent of the respondents. 
Table 1.4

Ethnoveterinary Medicinal Plants Utilized by Tribal Farmers of Attappadi to manage Livestock Production Diseases

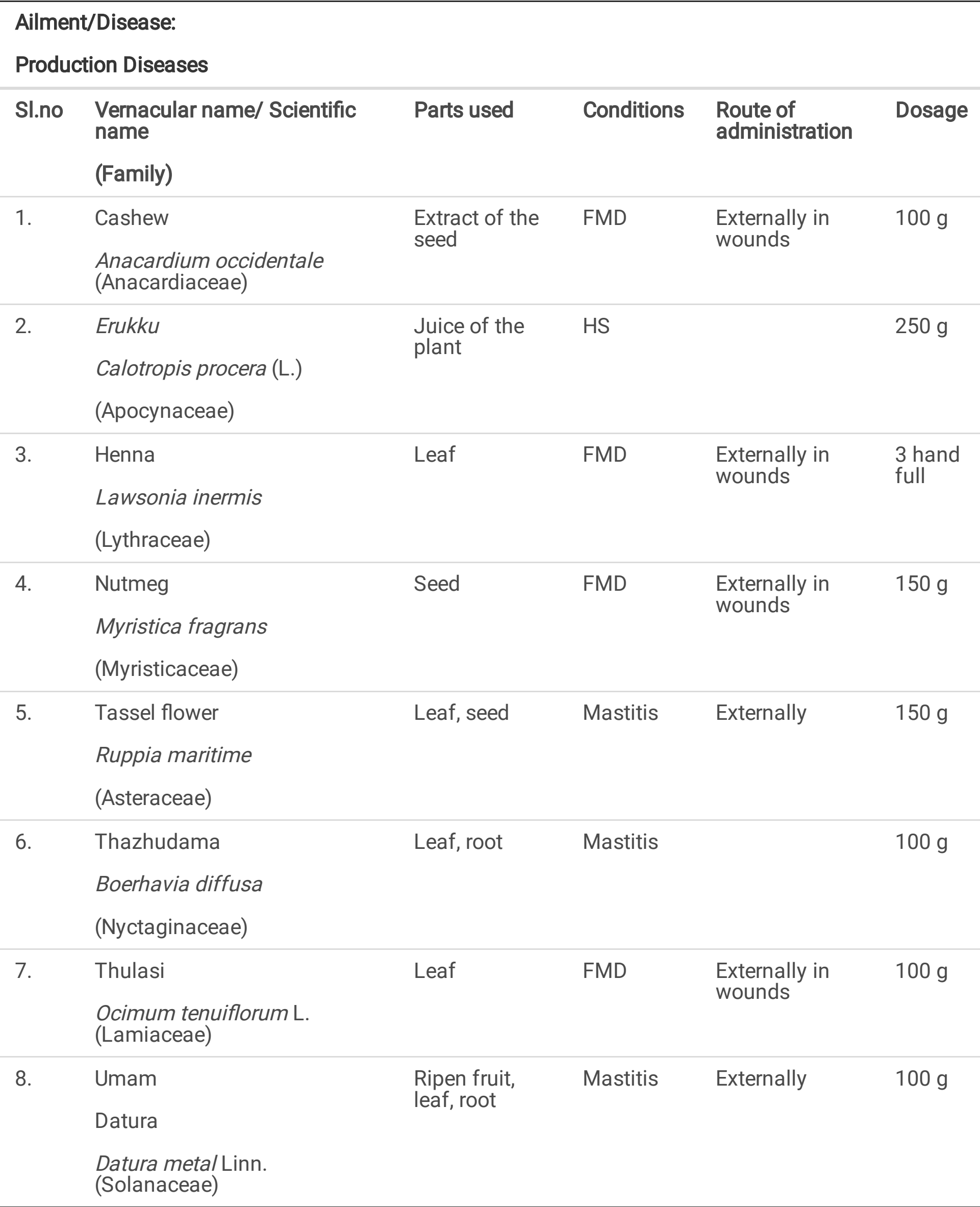


It could be observed from Table 1.4 that majority of the respondents $(73.47 \%)$ encountered mastitis with mixture paste of aloe vera $(250 \mathrm{~g})$, turmeric $(50 \mathrm{~g})$, calcium hydroxide $(10 \mathrm{~g})$ as external application.

Similarly, tarvine leaves, uncoated seeds of datura in fenugreek water were boiled in milk and was applied externally as paste. Tassel flower was used for rosy milk mastitis (11.22\%).

Touch me not leaves was ground and the paste was given orally for three days by 12.24 per cent of the respondents.

FMD was treated with paste made from cumin $(10 \mathrm{~g})$, fenugreek $(10 \mathrm{~g})$, turmeric $(10 \mathrm{~g})$, pepper $(10 \mathrm{~g})$, garlic (4 no.), jaggery (100g) with one coconut was given orally thrice a day by the 54.08 per cent of the respondents. Lard of wild boar in banana (89.80\%), veldt grape, curd $(0.75 \mathrm{I})$, with turmeric, neem leaves and red onion was given orally to counter FMD by 57.14 per cent of the respondents

The wound lesions were externally applied with thulasi leaves, henna leaves, neem leaves, turmeric, and garlic in coconut oil by 16.31 per cent of the respondents. Similarly, nutmeg oil, cashew oil was rubbed with hen feather in wound lesions $(12.24 \%)$. Turmeric powder with coconut oil and luke - warm water was applied to the lesion and was tied by 18.37 per cent of the respondents. Pox lesions in fowl and small ruminants were treated with neem leaves, turmeric in coconut oil by majority of the respondents $(59.18 \%)$.

Puncture vine and calotropis were used to treat inflammatory conditions. Edema lesions of HS were treated with paste of drumstick roots $(31.63 \%)$, lard of wild boar $(22.44 \%)$, calotropis and puncture vine leaves. 
Table 1.5

Ethnoveterinary Medicinal Plants Utilized by Tribal Farmers of Attappadi to manage Livestock Respiratory conditions

\begin{tabular}{|c|c|c|c|c|c|}
\hline \multicolumn{6}{|c|}{$\begin{array}{l}\text { Ailment/Disease: } \\
\text { Respiratory conditions }\end{array}$} \\
\hline SI.no & $\begin{array}{l}\text { Vernacular name/ } \\
\text { Scientific name } \\
\text { (Family) }\end{array}$ & Parts used & Conditions & $\begin{array}{l}\text { Route of } \\
\text { administration }\end{array}$ & Dosage \\
\hline 1. & $\begin{array}{l}\text { Ginger } \\
\text { Zingiber officinale } \\
\text { Rosc. } \\
\text { (Zingiberaceae) }\end{array}$ & Rhizome & $\begin{array}{l}\text { Respiratory } \\
\text { distress, Cough }\end{array}$ & Orally & $\begin{array}{l}50 \mathrm{~g}- \\
100 \mathrm{~g}\end{array}$ \\
\hline 2. & $\begin{array}{l}\text { Jeerakam } \\
\text { Cuminum cyminum } \\
\text { (Umbelliferae) }\end{array}$ & Fruit & Cough & Orally & $\begin{array}{l}50 \mathrm{~g}- \\
100 \mathrm{~g}\end{array}$ \\
\hline 3. & $\begin{array}{l}\text { Malabar nut } \\
\text { Justicia adhatoda } \\
\text { (Acanthaceae) }\end{array}$ & Leaf & $\begin{array}{l}\text { Respiratory } \\
\text { distress, Cough }\end{array}$ & Orally & $100 \mathrm{~g}$ \\
\hline 4. & $\begin{array}{l}\text { Neem } \\
\text { Azadirachta indica } \\
\text { (Meliaceae) }\end{array}$ & $\begin{array}{l}\text { Juice of the } \\
\text { plant, leaf }\end{array}$ & $\begin{array}{l}\text { Respiratory } \\
\text { distress }\end{array}$ & Inhalant & $250 \mathrm{~g}$ \\
\hline 5. & $\begin{array}{l}\text { Pepper } \\
\text { Piper nigrum L. } \\
\text { (Piperaceae) }\end{array}$ & Seed, leaf & Cough & Orally & $\begin{array}{l}50 \mathrm{~g}- \\
100 \mathrm{~g}\end{array}$ \\
\hline 6. & $\begin{array}{l}\text { Red onion } \\
\text { Allium sepa L. } \\
\text { (Liliaceae) }\end{array}$ & Bulb & Cough & Orally & $250 \mathrm{~g}$ \\
\hline 7. & $\begin{array}{l}\text { Thulasi } \\
\text { Ocimum tenuiflorum L. } \\
\text { (Lamiaceae) }\end{array}$ & Leaf & $\begin{array}{l}\text { Respiratory } \\
\text { distress }\end{array}$ & Inhalant & $250 \mathrm{~g}$ \\
\hline
\end{tabular}

The Table 1.5 denoted that Malabar nut with $50 \mathrm{~g}$ ginger, $200 \mathrm{~g}$ jaggery was given for 6 days in treatment for respiratory distress by 21.43 per cent of the respondents. Other followed practises included camphor vapour, neem leaves' vapour, ginger and thulasi leaves. Cough was given with mixture of $500 \mathrm{~g}$ ginger, 
$200 \mathrm{~g}$ jaggery (20.41\%) and Malabar nut for 6 days (14.29\%), and cuminum, pepper, red onion, zingiberis were given for fever by 20.41 per cent of the respondents. 
Table 1.6

Ethnoveterinary Medicinal Plants Utilized by Tribal Farmers of Attappadi to manage Livestock Skin \& Bone conditions

\section{Ailment/Disease:}

Skin \& Bone conditions

\begin{tabular}{|c|c|c|c|c|c|}
\hline Sl.no & $\begin{array}{l}\text { Vernacular name/ } \\
\text { Scientific name } \\
\text { (Family) }\end{array}$ & Parts used & Conditions & $\begin{array}{l}\text { Route of } \\
\text { administration }\end{array}$ & Dosage \\
\hline 1. & $\begin{array}{l}\text { Aatha } \\
\text { Sweetsop } \\
\text { Annona squamosa } \\
\text { (Magnoliales) }\end{array}$ & Leaf, fruit & Wounds, myasis & $\begin{array}{l}\text { External } \\
\text { application }\end{array}$ & $100 \mathrm{~g}$ \\
\hline 2. & $\begin{array}{l}\text { Cassia } \\
\text { Cassia fistula Linn. } \\
\text { (Fabaceae) }\end{array}$ & Seed & External parasites & $\begin{array}{l}\text { External } \\
\text { application }\end{array}$ & $100 \mathrm{~g}$ \\
\hline 3. & $\begin{array}{l}\text { Ficus } \\
\text { Ficus benghalensis } \\
\text { Linn (Moraceae) }\end{array}$ & Leaf, root & Myasis & $\begin{array}{l}\text { External } \\
\text { application }\end{array}$ & $100 \mathrm{~g}$ \\
\hline 4. & $\begin{array}{l}\text { Garlic } \\
\text { Allium sativum L. } \\
\text { (Amaryllidaceae) }\end{array}$ & Clove & warts, myasis & $\begin{array}{l}\text { External } \\
\text { application }\end{array}$ & 10 no. \\
\hline 5. & $\begin{array}{l}\text { Henna } \\
\text { Lawsonia inermis } \\
\text { (Lythraceae) }\end{array}$ & Leaf & Myasis & $\begin{array}{l}\text { External } \\
\text { application }\end{array}$ & $\begin{array}{l}3 \text { hand } \\
\text { full }\end{array}$ \\
\hline 6. & $\begin{array}{l}\text { Jeerakam } \\
\text { Cuminum cyminum } \\
\text { (Umbelliferae) }\end{array}$ & Fruit & warts, fracture & $\begin{array}{l}\text { External } \\
\text { application }\end{array}$ & $25 \mathrm{~g}$ \\
\hline 7. & $\begin{array}{l}\text { Kanjiram } \\
\text { Strychnine } \\
\text { Strychnos nux-vomica } \\
\text { Linn (Loganiaceae) }\end{array}$ & Root & Wounds & $\begin{array}{l}\text { External } \\
\text { application }\end{array}$ & $100 \mathrm{~g}$ \\
\hline
\end{tabular}




\section{Ailment/Disease:}

Skin \& Bone conditions

\begin{tabular}{|c|c|c|c|c|c|}
\hline 8. & $\begin{array}{l}\text { Mukkutti } \\
\text { Biophytum nervifolium } \\
\text { (Oxalidaceae) }\end{array}$ & Stem & wounds & $\begin{array}{l}\text { External } \\
\text { application }\end{array}$ & $150 \mathrm{~g}$ \\
\hline 9. & $\begin{array}{l}\text { Neem } \\
\text { Azadirachta indica } \\
\text { (Meliaceae) }\end{array}$ & $\begin{array}{l}\text { Juice of the } \\
\text { plant, leaf }\end{array}$ & $\begin{array}{l}\text { wounds, myasis, } \\
\text { external parasites, } \\
\text { Pox }\end{array}$ & $\begin{array}{l}\text { External } \\
\text { application }\end{array}$ & $150 \mathrm{~g}$ \\
\hline 10. & $\begin{array}{l}\text { Piranda } \\
\text { Cissus quadrangularis } \\
\text { (Vitaceae) }\end{array}$ & Stem, leaf & fracture & $\begin{array}{l}\text { As } \\
\text { immobilizer }\end{array}$ & $250 \mathrm{~g}$ \\
\hline 11. & $\begin{array}{l}\text { Thulasi } \\
\text { Ocimum tenuiflorum L. } \\
\text { (Lamiaceae) }\end{array}$ & Leaf & warts & $\begin{array}{l}\text { External } \\
\text { application }\end{array}$ & $100 \mathrm{~g}$ \\
\hline 12. & $\begin{array}{l}\text { Thumba } \\
\text { Leucas zeylanica } \\
\text { (Lamiaceae) }\end{array}$ & Leaf, stem & External parasites & $\begin{array}{l}\text { External } \\
\text { application }\end{array}$ & $500 \mathrm{~g}$ \\
\hline 13. & $\begin{array}{l}\text { Tobacco } \\
\text { Nicotiana tabacum } \\
\text { (Solanaceae) }\end{array}$ & $\begin{array}{l}\text { Leaf, } \\
\text { extract } \\
\text { from leaf }\end{array}$ & Wounds & $\begin{array}{l}\text { External } \\
\text { application }\end{array}$ & $500 \mathrm{~g}$ \\
\hline 14. & $\begin{array}{l}\text { Touch me not } \\
\text { Mimosa pudica } \\
\text { (Fabaceae) }\end{array}$ & $\begin{array}{l}\text { Leaf, root, } \\
\text { stem }\end{array}$ & wounds, sprain & $\begin{array}{l}\text { External } \\
\text { application }\end{array}$ & $250 \mathrm{~g}$ \\
\hline 15. & $\begin{array}{l}\text { Turmeric } \\
\text { Curcuma domestica } \\
\text { vallars (Zingiberaceae) }\end{array}$ & Rhizome & warts, myasis, Pox & $\begin{array}{l}\text { External } \\
\text { application }\end{array}$ & $100 \mathrm{~g}$ \\
\hline 16. & $\begin{array}{l}\text { Vatha kodi } \\
\text { Nervalia zeylanica } \\
\text { (Rununculaceae) }\end{array}$ & Leaf & Fracture & $\begin{array}{l}\text { External } \\
\text { application }\end{array}$ & $500 \mathrm{~g}$ \\
\hline
\end{tabular}

Table 1.6 indicates that Lacerated wounds were treated with touch me not plant leaves by 6.12 per cent of the respondents, sweetsop (8.16\%), strychnine roots in human urine by 6.12 per cent of the 
respondents and leaves of little tree plant with coconut oil (12.24\%). Umbilical wounds were treated with tobacco leaves and neem oil by 13.26 per cent of the respondents. Treatment of warts with mixture of garlic (10 no.), turmeric ( $10 \mathrm{~g})$, cumin $(25 \mathrm{~g})$, thulasi leaves and butter was followed by 21.42 per cent of the respondents.

For traumatic myasis, out of the six practises documented, ceylon leaves in slaked lime was followed by 16.33 per cent of the respondents, sweetsop leaves (8.16\%) and a mixture of neem leaves, turmeric $(20 \mathrm{~g})$, garlic, thulasi leaves and henna leaves $(18.37 \%)$ were applied for myasis. Saps of ficus in wick was sundried and were tied in maggot wounds by 8.16 per cent of the respondents. Young leaves of custard apple were impregnated to maggot wounds $(21.43 \%)$.

Ground mango leaves in goats' urine was given orally for 2-3 days for arthritis / joint pain (8.16\%). Majority of the farmers (48.97\%) used stem of veldt grape (3-4 no.) with cumin (a pinch) was made to a paste and was tied over the fracture site in animals and the lesion was externally fixed with stem of umbrella tree for four weeks. Sap of Vatha kodi with castor and chaff flower was used in fractures (33.67\%). Touch me not leaves were ground and paste was used in sprain conditions (58.16). 
Table 1.7

Ethnoveterinary Medicinal Plants Utilized by Tribal Farmers of Attappadi to manage Livestock General Health \& Poisoning conditions

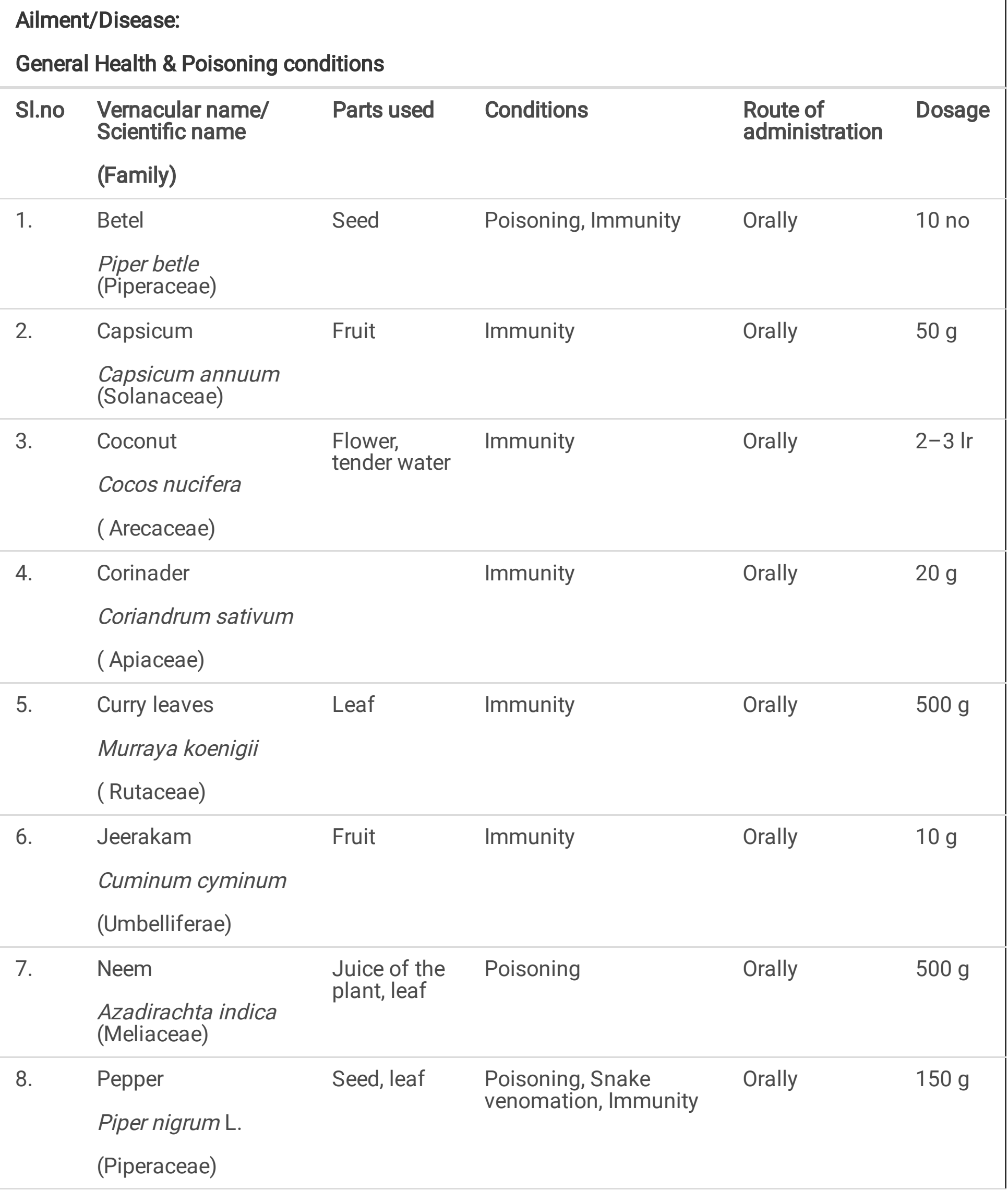




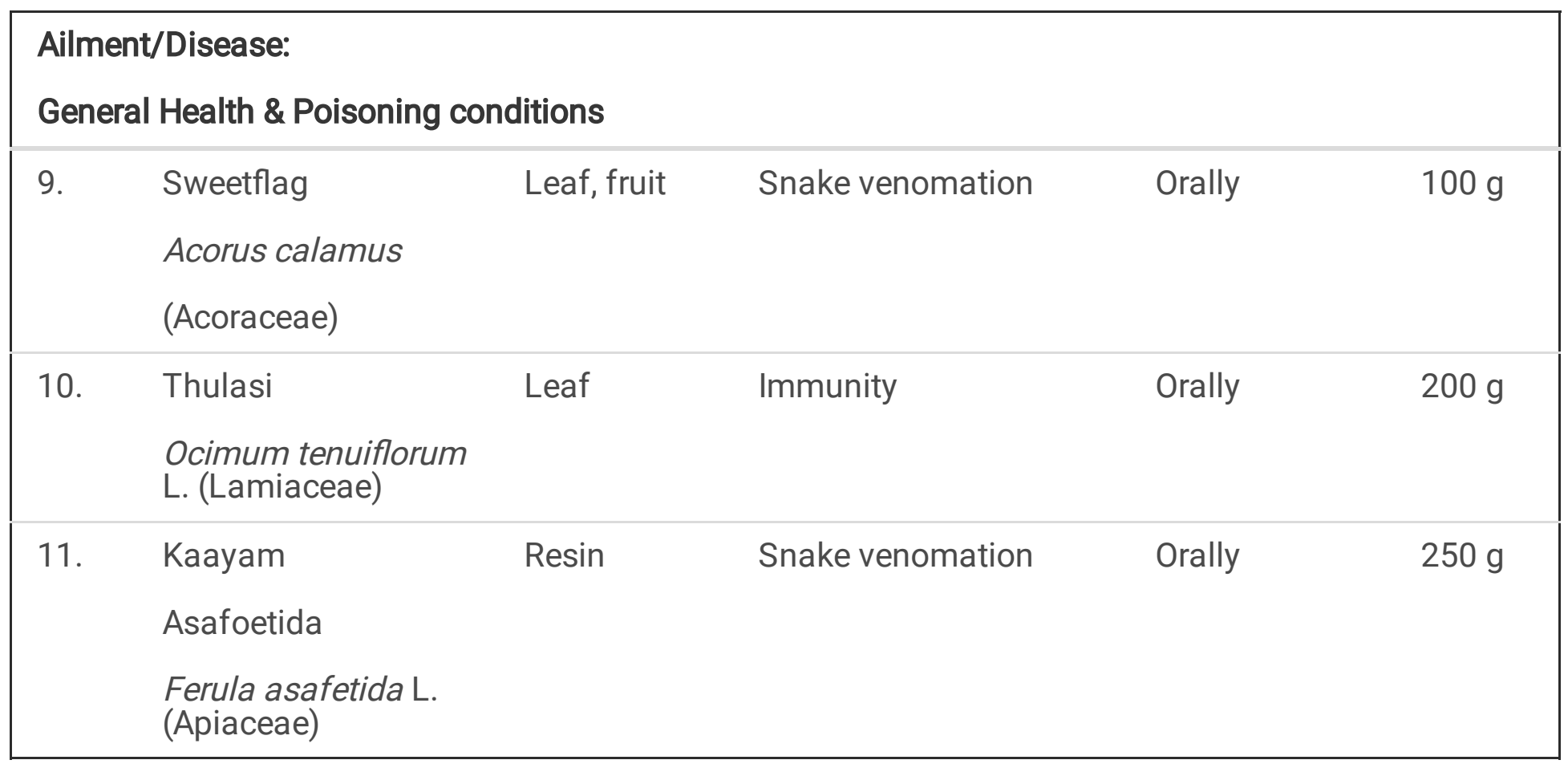

As the Table 1.7 depicts, in order improve milk production, boiled papaya, spinach leaves, muringa leaves and asparagus were given orally by $\mathbf{5 7 . 1 4}$ per cent of the respondents. Immunity was promoted using the combination of pepper $(10 \mathrm{~g})$, cumin $(10 \mathrm{~g})$, coriander $(20 \mathrm{~g})$, aloe vera $(100 \mathrm{~g})$, garlic $(50 \mathrm{~g})$, coconut, curry leaves, betel leaves, ginger $(50 \mathrm{~g})$, capsicum $(50 \mathrm{~g})$, thulasi leaves, jaggery $(100 \mathrm{~g})$ and rock salt.

Lard of wild boar (2.04\%), ground neem leaves (8.16\%) and a mixture of $10 \mathrm{~g}$ rock salt, 10 no. betel, 10 no. pepper was ground, and the paste was given orally for general poisoning conditions (12.24\%). Asafoetida, garlic, pepper and sweet flag was given orally for snake venomation by 16.33 per cent of the respondents.

\section{Conclusion}

The present work is one of the initial efforts to quantify the ethno medicinal information used in livestock health care in Attappadi that facilitates better option for the selection of widely used medicinal plants for searching and identifying bioactive compounds to treat ailments. The study reported 55 medicinal plants with their uses from the Attappadi area. The highlighted high $\mathrm{F}_{\mathrm{IC}}$ values in the present study have indicated that the species traditionally used to treat these ailments are worth searching for bioactive compounds and this has illuminated the cultural importance of this ethno veterinary wisdom which is consonantly blended with the tribal culture of the area. There is an urgent need to formulate suitable conservation strategies for naturally growing ethno medicinal plants to overcome their depletion from natural resources and to make these practices more eco- friendly.

\section{Ethics approval and consent to participate}

Not applicable 


\section{Consent for publication}

Prior and informed consent of local people's pictures had been obtained for publication.

\section{Declarations}

\section{Ethics approval and consent to participate}

Not applicable

\section{Consent for publication}

Prior and informed consent of local people's pictures had been obtained for publication.

\section{Availability of data and materials}

All data generated or analysed during this study are included in this published article

\section{Conflict of Interest}

None declared.

\section{Author Contributions}

All authors equally contributed.

\section{Funding}

No funding from any agencies.

\section{Acknowledgement}

The authors are grateful to the tribal livestock farmers of Attappadi tribal belt for willingness to share their valuable indigenous knowledge despite their hectic timings and thankful to Tamil Nadu Veterinary and Animal Sciences University for approving to conduct the study.

\section{References}

1. Dash S. Contribution of Livestock Sector to Indian Economy. Indian Journal of Research. 2017; 6(1).

2. Reddy V. Socio-Economic Status of Livestock Farmers of Ibrahimpur Village, North Goa District: A Benchmark Analysis, Economic Affairs. 2017; 62(2): 1-6.

3. Priyanka S \& Binita K. Importance of livestock sector in doubling farmers Income by 2022. Indian Journal of Economics and Development. 2017; 13(2a): 136-140. 
4. Bruchac, M. Indigenous Knowledge and Traditional Knowledge. In Smith, C. (Ed.), Encyclopedia of Global Archaeology. 2014:3814-3824. New York: Springer.

5. WHO monographs on selected medicinal plants. 2009. .Available at: https://www.who.int/medicines/areas/traditional/SelectMonoVol4.pdf

6. Census of India. Provisional population Total. Ministry of Home Affairs, Office of The Registrar and Census Commissioner, New Delhi, India. 2011.

7. Government of Kerala. Census Scheduled Tribes Development Department. 2001. Retrieved from https://kerala.gov.in/ scheduled-tribe-development.

8. Government of Kerala. Scheduled Tribes of Kerala at a glance. Retrieved from https://kirtads.kerala.gov.in/.

9. Trotter R T \& Logan M H, Informant census: A new approach for identifying potentially effective medicinal plants. In: Etkin, L.N. (Ed.), Plants in indigenous medicine and diet. (Redgrave, Bedford Hill, New York) 1986: 91-112.

10. Aziz, M.A., Khan, A.H., Adnan, M. et al.Traditional uses of medicinal plants used by Indigenous communities for veterinary practices at Bajaur Agency, Pakistan. J Ethnobiology Ethnomedicine. 2018: 14(11).

11. Ragupathy, S., Newmaster, S.G. Valorizing the 'Irulas' traditional knowledge of medicinal plants in the Kodiakkarai Reserve Forest, India. J Ethnobiology Ethnomedicine. 2009; 5(10).

12. Lulekal, E., Asfaw, Z., Kelbessa, E. et al.Ethnoveterinary plants of Ankober District, North Shewa Zone, Amhara Region, Ethiopia. J Ethnobiology Ethnomedicine. 2014; 10(21).

13. Ragupathy S, Steven N G, Maruthakkutti M, Velusamy B \& Muneer M et al, Consensus of the 'Malasars' traditional aboriginal knowledge of medicinal plants in the Velliangiri holy hills, India Journal of Ethnobiology and Ethnomedicine. 2008; 4(8).

14. Belayneh, A., Bussa, N.F. Ethnomedicinal plants used to treat human ailments in the prehistoric place of Harla and Dengego valleys, eastern Ethiopia. J Ethnobiology Ethnomedicine. 2014; 10(18).

15. Xiong, Y., Long, C. An ethnoveterinary study on medicinal plants used by the Buyi people in Southwest Guizhou, China. J Ethnobiology Ethnomedicine. 2020; 16(46).

16. Saha M R, Sarker D D \& Sen A., Ethnoveterinary practices among the tribal community of Malda district of West Bengal, India, Indian Journal of Traditional Knowledge. 2014; 13(2): 359- 367.

\section{Figures}




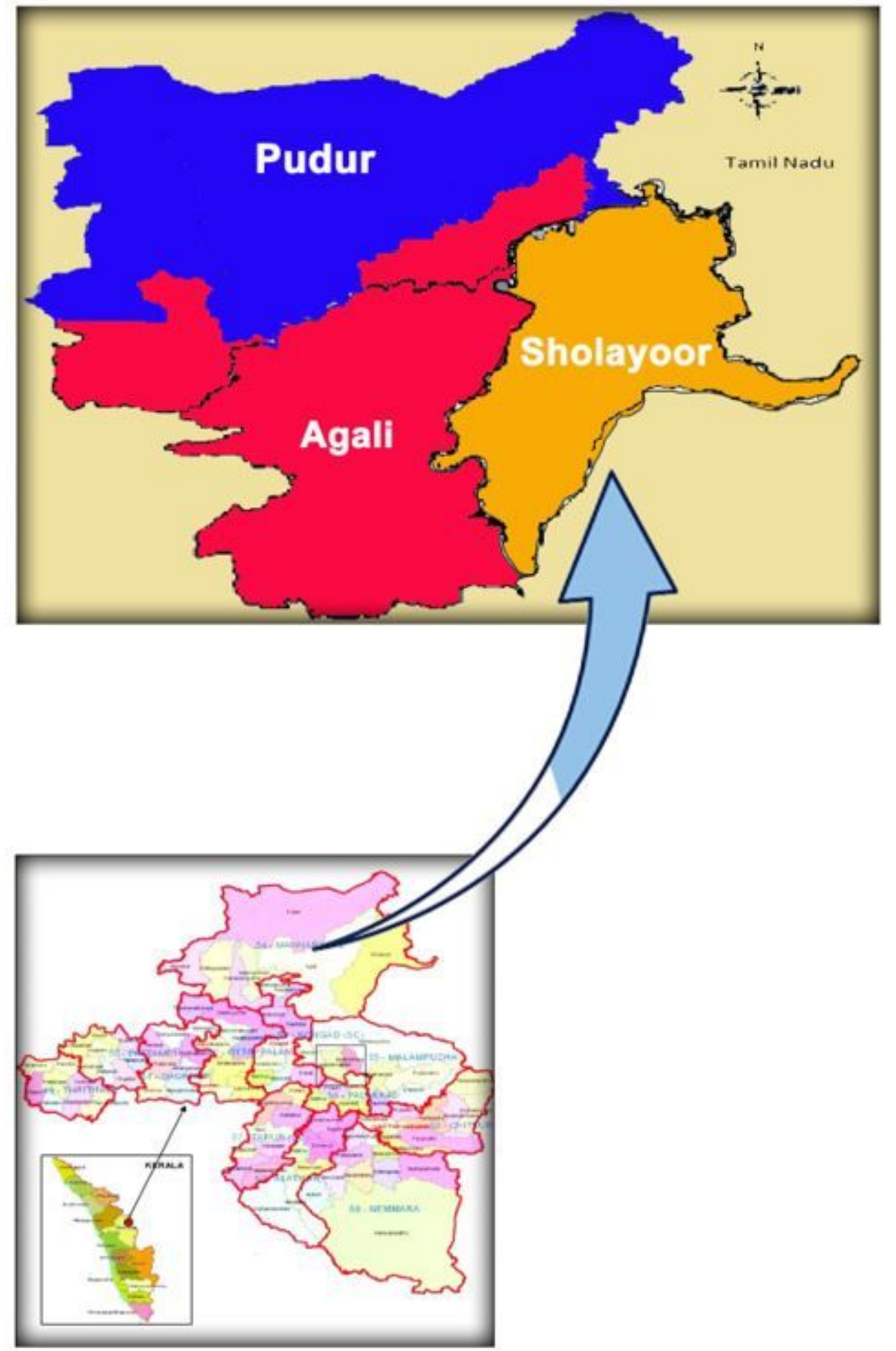

Figure 1

MAP SHOWING THE STUDY AREA 

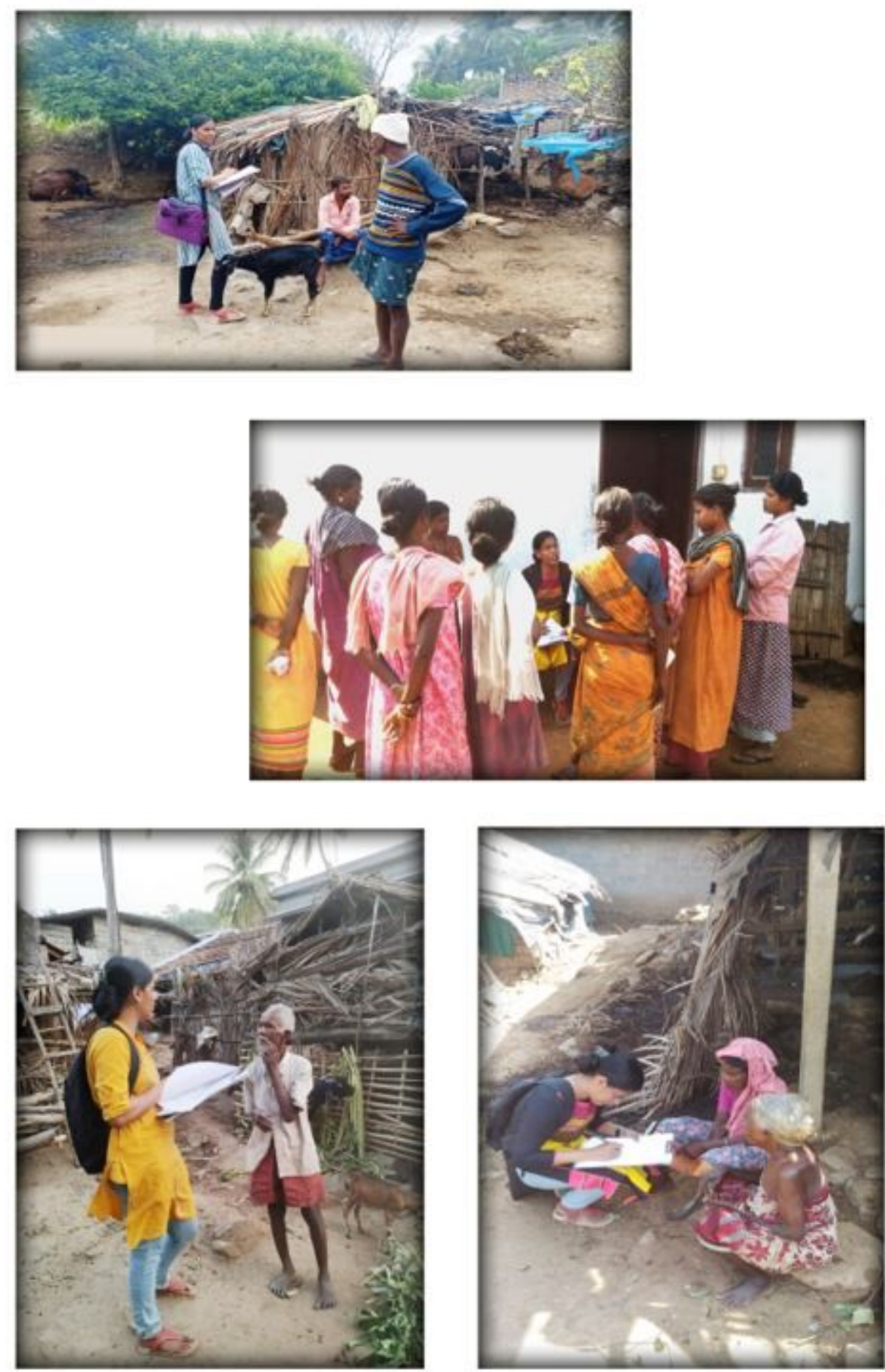

Figure 2

FIELD SURVEY AND PRIMARY DATA COLLECTION 

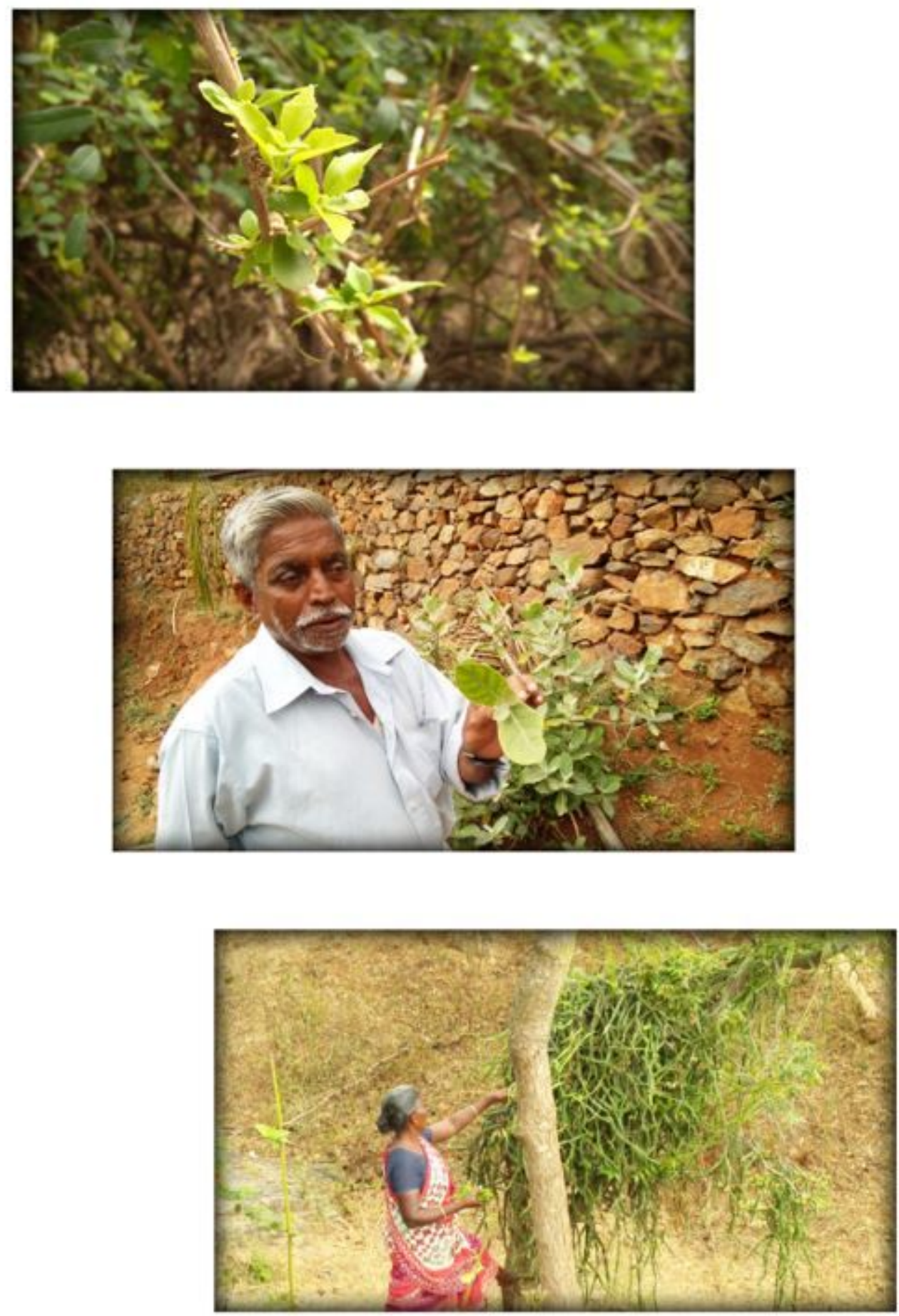

Figure 3

ETHNOVETERINARY MEDICINES USED BY THE ATTAPPADI TRIBAL FARMERS 